\title{
The research of Q-ary LDPC codes in the navigation system
}

\author{
$\mathrm{Xu} \mathrm{Yi}{ }^{1}$, Daoxing Guo ${ }^{2}$ and Wei You ${ }^{3}$ \\ College of Communications Engineering, PLA University of Science and Technology, Nanjing \\ 210007, China
}

Email: yixv084@163.com

Key words: Navigation system; Q-ary; LDPC; EMS; maximum information entropy

\begin{abstract}
In navigation system, due to the long distance between earth and satellites, the external environment noise may affect the service quality of information transmission system. To improve the capacity of anti-jam satellite communications, in this paper, an efficient construction algorithm for Q-ary LDPC code based on maximum information entropy is put forward, which brings extra gain to satellite communications system. The algorithm transforms the LDPC code check matrix $\mathrm{H}$ of the PEG method. By exchanging the "1" in the matrix with the generated element groups, the non-binary LDPC code $\mathrm{H}$ matrix is achieved. Simulation results demonstrate that the proposed algorithm achieves about $0.2 \mathrm{~dB}$ performance improvement compared with the random replacement method. Moreover, compared with the non-binary LDPC codes based on maximum information entropy exploited in GPS LDPC codes with the same code length and code rate, our proposed algorithm yields a better performance than that in GPS application scenarios.
\end{abstract}

\section{INTRODUCTION}

Navigation system can provide passive positioning, navigation, timing and other functions, its economic effect is prominent. At the same time Navigation system also plays an important role in safeguarding national security. At present global satellite navigation system in the world are the US GPS, Europe's Galileo, Russia's GLONASS and China's Beidou system.

In the navigation system, due to the distant star, the signal during transmission to the satellite link will be subject to greater interference. But the error correction code can provide a high coding gain to enhance the quality of service system and anti-jamming capability. Wherein the GPS navigation system uses a signal rate $1 / 2(1200,600)$ and $(548,274)$ binary LDPC to encode the signal;

The existing satellite navigation system has not been used Q-ary LDPC, therefore this article has a strong reference value.

\section{EMS decoding algorithm}

EMS decoding algorithm process is as follows:

1. Update variable node calculation

$$
U_{t p}\left[a_{1}, a_{2}, \cdots, a_{p}\right]=L\left[a_{1}, a_{2}, \cdots, a_{p}\right]+\sum_{v=1, v \neq t}^{d_{v}} V_{p v}\left[a_{1}, a_{2}, \cdots, a_{p}\right]
$$

2. Displacement step

$$
\begin{aligned}
& U_{p c}\left[a_{1}, a_{2}, \cdots, a_{p}\right]=U_{v p}\left[j_{1}, j_{2}, \cdots, j_{p}\right] \\
& a(X)=h(X) j(X)
\end{aligned}
$$

3. Update check node calculation

Update every check node needs to use this information in addition to the remaining $d_{c}-1$ variable nodes: $L\left(a_{k}\right)=\sum_{c=1, \cdots, d_{c}-1} u_{p c}^{\left(k_{c}\right)}$.Depending on the setting configuration set 


$$
\begin{gathered}
V_{d_{c} p}\left[a_{1}, a_{2}, \cdots, a_{p}\right]=\max _{a_{k} \in S_{i_{c}(x)}}\left\{L\left(a_{k}\right)\right\} \\
V_{c p}\left[a_{1}, a_{2}, \cdots, a_{p}\right]=V_{c p}\left[a_{1}, a_{2}, \cdots, a_{p}\right]-V_{c p}[0, \cdots, 0],\left(c=1 \ldots d_{c}\right)
\end{gathered}
$$

4. Inverse permutation step

$$
V_{p v}\left[a_{1}, a_{2}, \cdots, a_{p}\right]=V_{c p}\left[j_{1}, j_{2}, \cdots, j_{p}\right]
$$

$a(X)=h^{-1}(X) j(X)$.

5. Decoding judgment

$$
\hat{x}=\underset{a \in G F\left(2^{p}\right)}{\arg \max }\left\{L\left[a_{1}, a_{2}, \cdots, a_{p}\right]+\sum_{v=1}^{d_{v}} V_{p v}\left[a_{1}, a_{2}, \cdots, a_{p}\right]\right\}
$$

Complexity Analysis

In hardware implementation, the balance between performance and complexity should be taken into account[3].Decoding algorithm used in this project is EMS algorithm. The complexity of the EMS algorithm can be obtained by the following table:

Table 1 The computation iteration of EMS algorithm

\begin{tabular}{|r|c|c|}
\hline Iteration variable & addition & multiplication \\
\hline$L^{\prime}(m \rightarrow n)$ & $q+1+M\left(\sum_{i=1}^{n_{c}} C_{d_{c-1}}^{i} 1_{m}^{i}+C_{d_{c}-1}^{i} q\right)\left(d_{c}-1\right)$ & 0 \\
\hline$L^{\prime}(m \leftarrow n)$ & $N d_{v}^{2}(q-1)$ & 0 \\
\hline
\end{tabular}

Where $q$ denotes decimal, $M, N$ is rows and columns of the check matrix $H$, respectively. Row weight is $d_{c}$;Column weight is $d_{v}$.

There is not multiplication of EMS algorithm in Table 1,and with the value of $n_{m} n_{c}$ decreasing, the decoding complexity reduces.( $n_{m}$ and $n_{c}$ is configuration information of EMS algorithm).

\section{Construction of Q-ary LDPC}

Q-ary LDPC is defined in the finite field GF $(q),(q>2)$.Finite field arithmetic operation for closure. Finite element domain can be expressed as $G F\left(2^{m}\right)=\left\{0, \alpha^{0}, \alpha^{1}, \ldots, \alpha^{2^{m}-2}\right\}$, Finite element may be further determined by the primitive polynomial domain, and correspond to binary. Q-ary LDPC codes in practical applications are compatible binary information, which corresponds to each variable node is also connected to a plurality of small nodes, where each node represents a bit of information. As shown in Figure 1

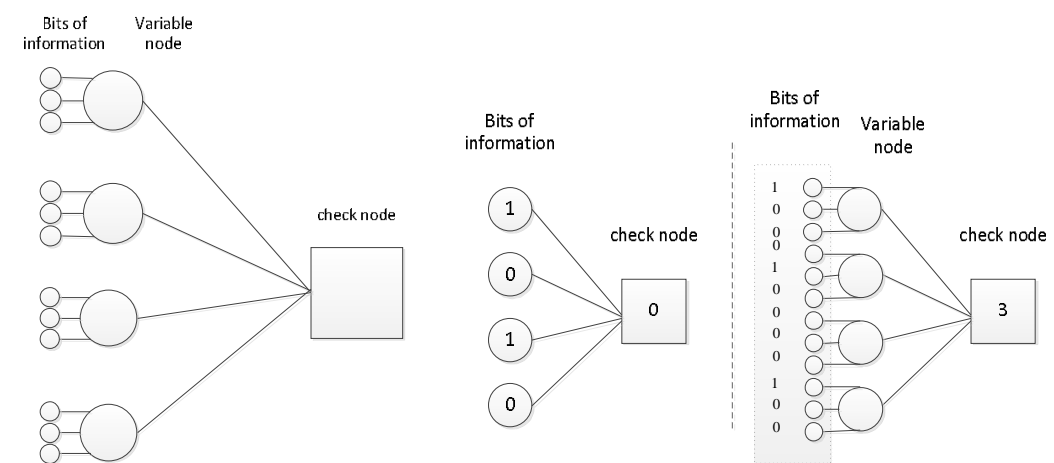

Figure 1 Representation of node of Q-ary LDPC and Error sensitivity of Q-ary LDPC

It is because of the structural characteristics of Q-ary LDPC, making binary LDPC codes compared to binary LDPC code has a better error sensitivity. As shown in Figure 2, The initial value of the variable nodes is all $0 \mathrm{~s}$, the variable connected to check nodes has two errors. Check nodes of 
binary LDPC can't check out error, but check nodes of Q-ary LDPC can check out by GF (q) addition. From Figure 2 can be drawn that Q-ary LDPC easier to find bit error and to correct it.

For the same code length, in the finite field GF (q),(q> 2), Q-ary LDPC code performance will be better than the binary. And the larger q, the greater the performance increase[4].This can be analyzed on the other hand: For LDPC code, if the check matrix H column weight is large enough, its performance can be arbitrarily close to Shannon limit[5].However, the column weight increase will lead to a sharp increase in the number of short loops in a bipartite graph, so that the decoding algorithm performance decline. The Q-ary LDPC code column weight can be increased without changing the bipartite graph, and thereby improve performance[6].

PEG method:

The basic idea of PEG method is to increase circle length of Tanner, in order to reduce the effects of feedback information .

$V=V_{C} \cup V_{V}$ is collection of nodes in the Tanner graph, $V_{V}=\left\{v_{1}, v_{2}, \ldots, v_{N}\right\}$ is representative of the set of check nodes, $C_{V}=\left\{c_{1}, c_{2}, \ldots, c_{M}\right\}$ is representative of the variable node set. $E=V_{C} \times V_{V}$ represents a collection of edges. As shown below, Figure 2 is a check matrix of simple Q-ary LDPC corresponding Tanner graph.

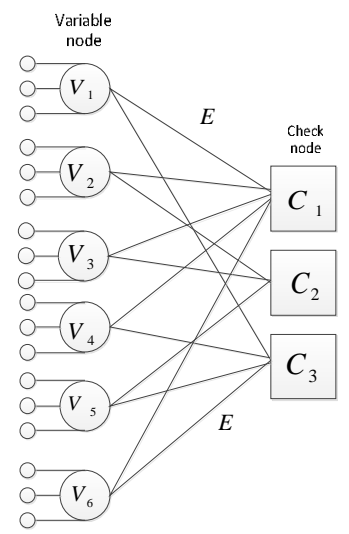

Figure 2 Tanner of Q-ary LDPC

Check node degree sequence is defined as $D_{c}=\left\{d_{c_{1}}, d_{c_{2}}, \ldots, d_{c_{M}}\right\}$;Variable node degree sequence is defined as $D_{v}=\left\{d_{v_{1}}, d_{v_{2}}, \ldots, d_{v_{N}}\right\}$.Elements of $D_{c}, D_{v}$ are arranged in ascending order. According to the variable node, the set of edges can be split to $E=E_{v_{1}} \cup E_{v_{2}} \ldots \cup E_{v_{N}} . E_{v_{j}}$ is representative of the set of edges that is connected to variable node in the Tanner graph. And $E_{v_{j}}^{k}$ is defined the k- th edge that connected to variable node of $v_{j}, 1 \leq k \leq d_{v_{j}}$. For a given variable node $v_{j}$. The collection of $N_{v_{j}}^{l}$ represents variable node $v_{j}$ after 1 iterations to reach the check node. $\overline{N_{v_{j}}^{l}}=V_{c} \backslash N_{v_{j}}^{l}$

The maximum entropy method can be divided into the following two steps: Firstly construct a standard binary check matrix based on the PEG method. Secondly, Design

group of elements based on maximum entropy method to replace the same line of heavy non-zero elements of a row vector[7].

In the first assume a particular channel model, such as binary symmetric channel. Then each element side information entropy is obtained based on the distribution matrix for different line $\mathrm{k}$ non-zero elements. Select the maximum entropy edge corresponding to non-zero elements group as non-zero elements of vector of rows in the parity check matrix .In this case, non-zero elements in each row in check matrix of binary LDPC is replaced by obtained elements group. Wherein, the greater the entropy side information, the better the performance of the decoder. Briefly, this method is essentially the maximum entropy side edges corresponding row vector as a line of check matrix. The following Tab. 2 shows the element group over GF (64) of different line weight. 
Table 2 Element group of line weight 4 and line weight 5line weight 5

\begin{tabular}{|c|c|c|c|c|c|c|}
\hline \multicolumn{3}{|c|}{ K=4, GF(64) } & \multicolumn{5}{c|}{ K=5, GF(64) } \\
\hline 131171 & 5922121 & 2823101 & 6222531 & 60452031 & 59444061 & 45211531 \\
\hline 5344241 & 5852401 & 1413111 & 58361371 & 52373131 & 25211171 & 34302791 \\
\hline
\end{tabular}

\section{Performance of Q-ary LDPC code}

The length of Q-ary LDPC code designed in this paper is 528 and 1200 respectively. Due to the low transmission rate of the navigation data in the system and taking into account the coding gain, we Generally use the coding efficiency of 0.5 of Q-ary LDPC code. The check matrix of dimension is $N / 2 * N$.Based on the above code length and coding efficiency, the designed optimization ary symbol size is about 6-7bits, The proposed Q-ary LDPC is over GF(64) GF(128). Taking into account the length of GF (64) code can be divisible by 6, facilitate encoding and decoding hardware implementation, ;most importantly its performance is better, we use Q-ary LDPC code over GF(64).

The designed Q-ary LDPC code in project is superior to the binary LDPC codes used in GPS, It obtains about $0.3 \mathrm{~dB}$ coding gain at BER of $10^{-6}$. Stop conditions for the simulation is error bits to 200 .

Q-ary LDPC code and binary LDPC code $(1200,600)$ is in the condition of the same code length and coding efficiency, their error correction performance show in Figure 3.

In BER $10^{-5}$, the Q-ary LDPC code is superior to GPS $(1200,600)$ code about $0.22 \mathrm{~dB}$;In BER $10^{-6}$, the Q-ary LDPC code is superior to GPS $(1200,600)$ code about $0.24 \mathrm{~dB}$.

Q-ary LDPC code compares with binary LDPC code GPS $(548,274)$, their error correction performance show in Figure 3.

In BER $10^{-5}$, the Q-ary LDPC code is superior to GPS $(548,274)$ code about $0.25 \mathrm{~dB}$;In BER $10^{-6}$, the Q-ary LDPC code is superior to GPS $(1200,600)$ code about $0.3 \mathrm{~dB}$.
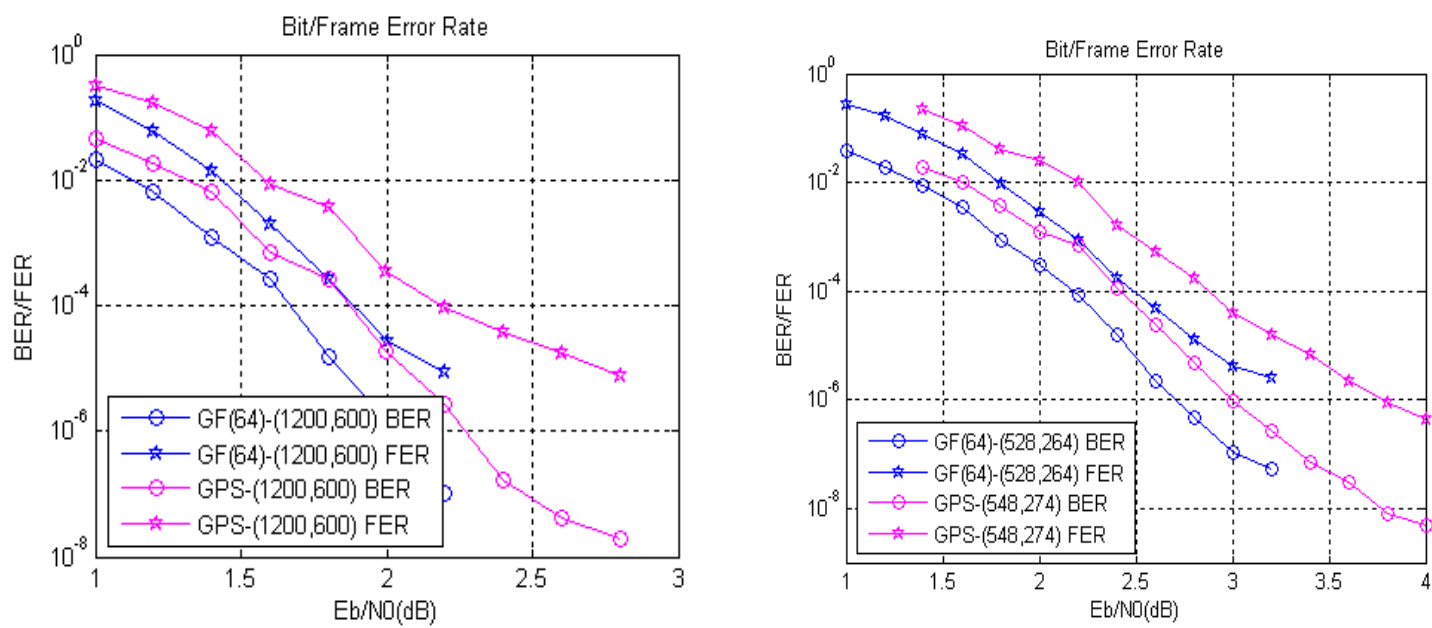

Figure 3 Error performance of code length 1200 and code length 528

\section{CONCLUSIONS}

Based on the practical demand of the current satellite navigation systems, we pursue a comprehensive analysis of the construction algorithm of Q-ary LDPC suitable for navigation signal system. The maximum entropy method is explored to construct the superior performance code, and the performance of the proposed Q-ary LDPC code and error correction code of GPS are compared. With the rapid development of digital signal process (DSP) technique, the hardware implementation complexity of Q-ary LDPC code will obtain significantly reduction, which leverages the application of Q-ary LDPC code in satellite navigation system and thus achieves a 
satisfactory performance.

\section{REFERENCES}

[1] D.Declercq and M.Fossorier,"Decoding algorithms for nonbinary LDPC codes over GF(q)," submitted to IEEE Trans. Commun.,2005.

[2] Voicila, D.Declercq, F.Verdier, M.Fossorier, P.Urard, "Low-complexity, Low-memory EMS algorithm for non-binary LDPC codes", in Proceedings of IEEE Int.Conf. Commun, ICC'2007,pp. 671-676, Glasglow,UK, June 2007

[3] L.Barnault and D.Declercq, "Fast decoding algorithm for LDPC over GF(2q)," in Proc. ITW2003, pp.70-73, Paris,France,Mar.2003.

[4] Z.-W.Li, L.Chen, L.-Q.Zeng, S.Lin, and W.Fong, "Efficient encoding of quasi-cyclic low-density parity-check codes," IEEE Trans. Commun, vol.54., pp.71-81, Jan.2006.

[5] Y.Y.Tai, L.Lan, L.Zeng, S.Lin, and K.Abdel-Ghaffar, "Algebraic construction of quasi-cyclic LDPC codes for the AWGN and erasure channels," IEEE Trans. Commun., vol.54,no. 10,pp. 1765- 1774,Oct.2006.

[6] L.Lan, Y.Y.Tai, S.Lin, B.Memari, and B.Honary, New constructions of quasi-cyclic LDPC codes based on special classes of BIBDs for the AWGN and binary erasure channels, IEEE Trans. Commun., 2008, 56(1): 39-48.

[7] A.R.Iyengar,M.Papaleo,P.H.Siegel,J.K.Wolf,A.Vanelli-Coralli,And G.E.Corazza,'Windowed decoding of protograph-based LDPC convolutional codes over erasure channels'IEEE Trans,Inf.Theory,vol.58,no.4,pp.2303-2320.2012 